Tohoku Math. J.

58 (2006), 237-258

\title{
BOREL SUMMABILITY OF DIVERGENT SOLUTIONS FOR SINGULARLY PERTURBED FIRST-ORDER ORDINARY DIFFERENTIAL EQUATIONS
}

\author{
MASAKI HIBINO
}

(Received May 26, 2004, revised March 8, 2005)

\begin{abstract}
This paper is concerned with the study of the Borel summability of divergent solutions for singularly perturbed inhomogeneous first-order linear ordinary differential equations which have a regularity at the origin. In order to assure the Borel summability of divergent solutions, global analytic continuation properties for coefficients are required despite the fact that the domain of the Borel sum is local.
\end{abstract}

1. Introduction and main result. In this paper we are concerned with the following inhomogeneous first-order linear ordinary differential equation with a parameter $\varepsilon(\in \boldsymbol{C})$ :

$$
a(x, \varepsilon) D_{x} u(x, \varepsilon)+b(x, \varepsilon) u(x, \varepsilon)=f(x, \varepsilon),
$$

where $x \in C, D_{x}=d / d x$. The coefficients $a, b$ and $f$ are holomorphic at $(x, \varepsilon)=(0,0)$ $\in C^{2}$.

First of all we give two fundamental assumptions. The first means that $\varepsilon$ is a perturbation parameter; that is, we assume the following:

$$
a(x, 0) \equiv 0 \text {. }
$$

The second is

$$
a_{\varepsilon}(0,0) \neq 0,
$$

where $a_{\varepsilon}(x, \varepsilon)=(\partial / \partial \varepsilon) a(x, \varepsilon)$. These two assumptions imply that $a(0, \varepsilon) \neq 0$ for sufficiently small $\varepsilon \neq 0$, which means that (1.1) has a regularity at $x=0$.

Throughout this paper we always assume (1.2) and (1.3).

It follows from (1.2) and (1.3) that solutions of (1.1) can be expressed by convergent power series around $x=0$. Here, however, let us consider solutions expressed by power series in the perturbation parameter $\varepsilon$. Then, as will be stated later, under a suitable condition (1.1) has a unique power series solution $u(x, \varepsilon)=\sum_{n=0}^{\infty} u_{n}(x) \varepsilon^{n}\left(u_{n}(x)\right.$ are holomorphic in a common neighborhood of $x=0$ ), but it diverges in general and the rate of divergence is characterized in terms of the Gevrey index (cf. Definition 1.1 and Theorem 1.1).

So, we would like to study the following problem:

Is there any holomorphic solution $U(x, \varepsilon)$ which has the divergent solution $u(x, \varepsilon)$ as an asymptotic expansion of the Gevrey type when $\varepsilon \rightarrow 0$ ?

2000 Mathematics Subject Classification. Primary 35C20; Secondary 35C10, 35C15.

Key words and phrases. Singular perturbation, divergent solution, Borel summability, analytic continuation. 
We have two types of the Gevrey asymptotic expansions: 'asymptotic expansions in narrow regions' and 'asymptotic expansions in wide regions'. On the first, the above problem has been already studied by Hibino [8] (cf. Theorem 1.3). Therefore, the subject matter of this paper is the second expansion, and in particular we are interested in the Borel summability of the divergent solution stated above (cf. Definition 1.3). Our main purpose in this paper is to obtain the conditions under which the divergent solution is Borel summable.

The content of this paper is as follows. In Section 1.1 we state the condition which (1.1) should satisfy in order to assure the unique existence of the power series solution $u(x, \varepsilon)=$ $\sum_{n=0}^{\infty} u_{n}(x) \varepsilon^{n}$. Moreover, we give the rate of divergence, that is, the Gevrey index of $u(x, \varepsilon)$, and explain that this Gevrey index is optimal by investigating a simple example. This result (Theorem 1.1) has been proved by Hibino [7] and it plays the most fundamental role throughout this paper. In Section 1.2 we briefly explain the Gevrey asymptotic theory in narrow regions. As will be stated in Theorem 1.3, when the region is narrow, there always exists a holomorphic solution $U(x, \varepsilon)$ on that region which has the above divergent solution $u(x, \varepsilon)$ as an asymptotic expansion of the Gevrey type, without any additional condition for coefficients. This result has been proved in [8]. In Section 1.3 we give the main result in this paper (Theorem 1.5). When the region is wide, we cannot unconditionally expect the existence of $U(x, \varepsilon)$ stated above. In Theorem 1.5 we give the conditions under which such a $U(x, \varepsilon)$ exists. Global analytic continuation properties for the coefficients will be required. In Section 1.4 we introduce literature studying related topics. The proof of Theorem 1.5 is done through Sections 2, 3 and 4. In Section 2, the proof of Theorem 1.5 is reduced to that of a global solvability of the initial value problem of some convolution equation. Sections 3 and 4 constitute the main part of the proof. We transform the convolution equation obtained in Section 2 into some integral equation, and prove the global solvability of that integral equation by applying an iteration method. In Section 5, we investigate some special cases in detail. When an equation has a restricted form, we can obtain a stricter result than Theorem 1.5. Through these considerations, we will see the essentialness of our global assumptions.

1.1. Existence of a divergent solution. In this section we state the theorem obtained in [7], which assures the unique existence of divergent power series solution. First, let us give the definition of divergent power series of the Gevrey type.

DEFINITION 1.1. (1) $\mathcal{O}[R]$ denotes the ring of holomorphic functions on the closed ball $B(R)=\{x \in C ;|x| \leq R\}$, where $R$ is a positive number.

(2) The ring of formal power series in $\varepsilon(\in \boldsymbol{C})$ over the ring $\mathcal{O}[R]$ is denoted as $\mathcal{O}[R][[\varepsilon]]:$

$$
\mathcal{O}[R][[\varepsilon]]=\left\{u(x, \varepsilon)=\sum_{n=0}^{\infty} u_{n}(x) \varepsilon^{n} ; u_{n}(x) \in \mathcal{O}[R]\right\} .
$$

(3) We say that $u(x, \varepsilon)=\sum_{n=0}^{\infty} u_{n}(x) \varepsilon^{n}(\in \mathcal{O}[R][[\varepsilon]])$ belongs to $\mathcal{O}[R][[\varepsilon]]_{2}$, if there exist some positive constants $C$ and $K$ such that 


$$
\max _{|x| \leq R}\left|u_{n}(x)\right| \leq C K^{n} n !
$$

for all $n=0,1,2, \ldots$. The suffix 2 of $\mathcal{O}[R][[\varepsilon]]_{2}$ expresses the Gevrey index of power series. Elements of $\mathcal{O}[R][[\varepsilon]]_{2}$ are divergent power series in general.

Now we already know the following theorem, which will be fundamental in the argument below.

THEOREM 1.1 ([7]). Let us assume $b(0,0) \neq 0$. Then (1.1) has a unique power series solution $u(x, \varepsilon)=\sum_{n=0}^{\infty} u_{n}(x) \varepsilon^{n} \in \mathcal{O}[R][[\varepsilon]]_{2}$ for some $R>0$.

In the following we always assume $b(0,0) \neq 0$. On the basis of Theorem 1.1, we can study the coming problem; that is, the existence of asymptotic solutions of Gevrey type.

REMARK 1.1. The Gevrey index 2 of power series solutions $u(x, \varepsilon)$ (that is, the estimates (1.5)) is optimal. For example, let us consider the following simple equation:

$$
-\varepsilon D_{x} u(x, \varepsilon)+u(x, \varepsilon)=f(x) .
$$

Equation (1.6) has a unique power series solution $u(x, \varepsilon)=\sum_{n=0}^{\infty} f^{(n)}(x) \varepsilon^{n}$. Hence, if $f(x)=1 /(1-x)$ for example, it holds that $u_{n}(x) \equiv f^{(n)}(x)=n ! /(1-x)^{n+1}$. Therefore, in this case, the Gevrey index of $u(x, \varepsilon)$ is exactly 2 .

1.2. Gevrey asymptotic expansions in narrow regions. In this section we explain the result obtained in [8]. First we give the definition of the Gevrey asymptotic expansions in sectors.

Definition 1.2. (1) For $\theta \in \boldsymbol{R}, p>0$ and $0<\rho \leq+\infty$, the sector $S(\theta, p, \rho)$ in the universal covering space of $\boldsymbol{C} \backslash\{0\}$ is defined by

$$
S(\theta, p, \rho)=\left\{\varepsilon ;|\arg (\varepsilon)-\theta|<\frac{p}{2}, 0<|\varepsilon|<\rho\right\} .
$$

We refer to $\theta, p$ and $\rho$ as the bisecting direction, the opening angle and the radius of $S(\theta, p, \rho)$, respectively.

(2) Let $u(x, \varepsilon)=\sum_{n=0}^{\infty} u_{n}(x) \varepsilon^{n} \in \mathcal{O}[R][[\varepsilon]]_{2}$ and let $U(x, \varepsilon)$ be a holomorphic function on $X=B(R) \times S(\theta, p, \rho)$. Then we say that $U(x, \varepsilon)$ has $u(x, \varepsilon)$ as an asymptotic expansion of the Gevrey order 2 in $X$ if the following asymptotic estimates hold: there exist some positive constants $C$ and $K$ such that

$$
\max _{|x| \leq R}\left|U(x, \varepsilon)-\sum_{n=0}^{N-1} u_{n}(x) \varepsilon^{n}\right| \leq C K^{N} N !|\varepsilon|^{N},
$$

for all $\varepsilon \in S(\theta, p, \rho)$ and $N=1,2, \ldots$ Then we write this as

$$
U(x, \varepsilon) \cong_{2} u(x, \varepsilon) \text { in } X .
$$

The following result is well known as the theorem of Borel-Ritt. 
THEOREM 1.2 (cf. Balser [1,2]). Let $u(x, \varepsilon)=\sum_{n=0}^{\infty} u_{n}(x) \varepsilon^{n}$ be an arbitrary element of $\mathcal{O}[R][[\varepsilon]]_{2}$ and let $\theta$ be an arbitrary real number. Let us assume $0<p<\pi$. Then there always exist infinitely many holomorphic functions $U(x, \varepsilon)$ on some $X=B(r) \times$ $S(\theta, p, \rho)$ satisfying $U(x, \varepsilon) \cong_{2} u(x, \varepsilon)$ in $X$.

Now let $u(x, \varepsilon)=\sum_{n=0}^{\infty} u_{n}(x) \varepsilon^{n} \in \mathcal{O}[R][[\varepsilon]]_{2}$ be the divergent solution of (1.1). We recall that the unique existence of such a $u(x, \varepsilon)$ is ensured by Theorem 1.1. For a given bisecting direction $\theta$ and a given opening angle $p$, we consider the following problem: is there a holomorphic solution $U(x, \varepsilon)$ on $X=B(r) \times S(\theta, p, \rho)$ (for some $r$ and $\rho$ ) which satisfies $U(x, \varepsilon) \cong_{2} u(x, \varepsilon)$ in $X$ ? As an application of Theorem 1.2, in [8] it was proved that this problem was solved positively if $p<\pi$.

THEOREM 1.3 ([8]). Let $u(x, \varepsilon)=\sum_{n=0}^{\infty} u_{n}(x) \varepsilon^{n} \in \mathcal{O}[R][[\varepsilon]]_{2}$ be the divergent solution of (1.1), and let $\theta$ be an arbitrary real number. Let us assume $0<p<\pi$. Then there exist some positive constants $r_{p}, \rho_{p}$ and a holomorphic solution $U(x, \varepsilon)$ of $(1.1)$ on $X_{p}=B\left(r_{p}\right) \times S\left(\theta, p, \rho_{p}\right)$ such that $U(x, \varepsilon) \cong 2 u(x, \varepsilon)$ in $X_{p}$. Moreover, there are infinitely many such solutions.

The assumption $p<\pi$ is significant. Moreover, it should be remarked that we impose no additional conditions for coefficients. If a region becomes wider, we cannot unconditionally expect the existence of such an asymptotic solution $U(x, \varepsilon)$ as stated in Theorem 1.3. However, if it exists, then we see that it is unique from a general theory of the Gevrey asymptotic expansions (cf. Balser [1, 2], Lutz et al. [11] and Malgrange [12]). So, when does it exist? The main purpose of this paper is to answer this question in the case where the region is an open disk (on the precise definition, see Definition 1.3). Before giving the answer for the general equation (1.1), here let us try considering the special equation (1.6).

We recall that the divergent solution of (1.6) is given by $u(x, \varepsilon)=\sum_{n=0}^{\infty} f^{(n)}(x) \varepsilon^{n}$, and remark that the formal Borel transform $\mathcal{B}(u)(x, \eta)$ (cf. Definition 2.1) of $u(x, \varepsilon)$ is given by $\mathcal{B}(u)(x, \eta)=\sum_{n=0}^{\infty} f^{(n)}(x) \eta^{n} / n !=f(x+\eta)$. Then it follows from Theorems 1.4 and 2.1 that $U(x, \varepsilon)$ exists if and only if $f(x)$ can be continued analytically along the half line $\boldsymbol{R}_{+} e^{i \theta}=\left\{r e^{i \theta} ; r \geq 0\right\}$ and it has a exponential growth estimate $|f(x)| \leq C e^{\delta|x|}$. Moreover, $U(x, \varepsilon)$ can be represented explicitly by the formula

$$
U(x, \varepsilon)=\frac{1}{\varepsilon} \int_{\boldsymbol{R}_{+} e^{i \theta}} e^{-\eta / \varepsilon} f(x+\eta) d \eta .
$$

Consequently, we see, for example, the following facts.

(i) If $f(x)=1 /(1-x)$, then $U(x, \varepsilon)$ exists for all $\theta$ except $\theta=0$. For $\theta=0, U(x, \varepsilon)$ never exists.

(ii) If the existence region of $f(x)$ is a bounded one containing the origin, then $U(x, \varepsilon)$ by no means exists regardless of the choice of $\theta$.

The above unique asymptotic solution $U(x, \varepsilon)$ is called the Borel sum of $u(x, \varepsilon)$. In the next section, we give the conditions which the coefficients of (1.1) should satisfy in order to 
make certain the existence of the Borel sum. As we see from the above example, there is a close affinity between the existence of the Borel sum and global properties of coefficients.

1.3. Main result. First we give the precise definition of the Borel sum.

Definition 1.3. (1) For $\theta \in \boldsymbol{R}$ and $T>0$, we define the region $O(\theta, T)$ by

$$
O(\theta, T)=\left\{\varepsilon ;\left|\varepsilon-T e^{i \theta}\right|<T\right\} .
$$

(2) Let $u(x, \varepsilon)=\sum_{n=0}^{\infty} u_{n}(x) \varepsilon^{n} \in \mathcal{O}[R][[\varepsilon]]_{2}$. We say that $u(x, \varepsilon)$ is Borel summable in a direction $\theta$ if there exists a holomorphic function $U(x, \varepsilon)$ on $\mathcal{X}=B(r) \times O(\theta, T)$ for some $0<r \leq R$ and $T>0$ which satisfies $U(x, \varepsilon) \cong_{2} u(x, \varepsilon)$ in $\mathcal{X}$; that is, there exist some positive constants $C$ and $K$ satisfying the asymptotic estimates $\max _{|x| \leq r} \mid U(x, \varepsilon)-$ $\left.\sum_{n=0}^{N-1} u_{n}(x) \varepsilon^{n}\left|\leq C K^{N} N !\right| \varepsilon\right|^{N}$ for all $\varepsilon \in O(\theta, T)$ and $N=1,2, \ldots$.

We remark that in the case $0<p<\pi$, for any $O(\theta, T)$ it holds that $S(\theta, p, \rho) \subset$ $O(\theta, T)$ by taking a suitable $\rho>0$. In this sense, $O(\theta, T)$ is wider than a sector with an opening angle less than $\pi$. Because of this wide property, a given divergent power series $u(x, \varepsilon) \in \mathcal{O}[R][[\varepsilon]]_{2}$ is not necessarily Borel summable in general. (Compare with Theorem 1.2. In Theorem 2.1, we give the necessary and sufficient condition under which a given $u(x, \varepsilon)$ is Borel summable.) However, as was mentioned in Section 1.2, when $u(x, \varepsilon)$ is Borel summable in a direction $\theta$, the above holomorphic function $U(x, \varepsilon)$ is unique. So we call this unique $U(x, \varepsilon)$ the Borel sum of $u(x, \varepsilon)$ in a direction $\theta$.

The problem of the present paper is the existence of a holomorphic solution $U(x, \varepsilon)$ satisfying $U(x, \varepsilon) \cong_{2} u(x, \varepsilon)$ in $\mathcal{X}$. Let us divide the problem into the following two parts.

(I) When is $u(x, \varepsilon)$ Borel summable?

(II) Is the Borel sum $U(x, \varepsilon)$ a solution?

By the following theorem, problem (II) is always solved affirmatively. This is an immediate consequence of the uniqueness of the Borel sum.

THEOREM 1.4 (cf. Hibino [10]). Let $u(x, \varepsilon)=\sum_{n=0}^{\infty} u_{n}(x) \varepsilon^{n} \in \mathcal{O}[R][[\varepsilon]]_{2}$ be the divergent solution of $(1.1)$, and let us assume that $u(x, \varepsilon)$ is Borel summable in some direction $\theta$. Then its Borel sum $U(x, \varepsilon)$ is a holomorphic solution of (1.1).

In the remainder of this paper, we consider problem (I). To begin with, let us rewrite (1.1) to state the main result. By the condition $b(0,0) \neq 0$, we see that $b(x, 0) \neq 0$ in a neighborhood of $x=0$. Hence, by dividing both sides of (1.1) by $b(x, 0)$, we may assume that $b(x, 0) \equiv 1$. Then it follows from (1.2) and (1.3) that (1.1) is rewritten in the following form:

$$
\{\alpha(x)+\beta(x, \varepsilon)\} \varepsilon D_{x} u(x, \varepsilon)+\{1+\gamma(x, \varepsilon) \varepsilon\} u(x, \varepsilon)=f(x, \varepsilon),
$$

where each coefficient is holomorphic at the origin. Moreover $\alpha$ and $\beta$ satisfy

$$
\begin{gathered}
\alpha(0) \neq 0, \\
\beta(x, 0) \equiv 0 .
\end{gathered}
$$


Now let us give additional conditions which the coefficients should satisfy in order to assure the Borel summability of the divergent solution in a given direction $\theta$.

Assumptions. First we state the assumption for $\alpha(x)$. Let us consider the following initial value problem:

$$
\frac{d x}{d \xi}=-\alpha(x), \quad x(0)=0
$$

(A1) We assume the following:

(i) (1.13) has a holomorphic solution $x=\chi(\xi)$ on $E_{+}(\theta, \kappa)$ for some $\kappa>0$;

(ii) $(d \chi / d \xi)(\xi) \neq 0$ for $\xi \in E_{+}(\theta, \kappa)$.

Here $E_{+}(\theta, \kappa)(\kappa>0)$ is the region defined by

$$
E_{+}(\theta, \kappa)=\left\{\xi ; \operatorname{dist}\left(\xi, \boldsymbol{R}_{+} e^{i \theta}\right) \equiv \inf \left\{|\xi-\zeta| ; \zeta \in \boldsymbol{R}_{+} e^{i \theta}\right\} \leq \kappa\right\} .
$$

It is obvious that $\chi(\xi)$ is unique, if it exists.

Next, in order to state the assumptions for the other coefficients, we introduce the notation. First, let us define the region $\Omega_{\theta, \kappa}$ consisting of solution curves of (1.13) by

$$
\Omega_{\theta, \kappa}=\left\{\chi(\xi) ; \xi \in E_{+}(\theta, \kappa)\right\} .
$$

The assumption (A1) implies that $\alpha(x)$ is analytic on $\Omega_{\theta, \kappa}$ and that $\alpha(x) \neq 0$ for all $x \in \Omega_{\theta, \kappa}$. Secondly, let us define the function $A(x)$ on $\Omega_{\theta, \kappa}$ by

$$
A(x)=-\int_{0}^{x} \frac{d z}{\alpha(z)}, \quad x \in \Omega_{\theta, \kappa} .
$$

Here the path of integration is the solution curve of (1.13). Then $A(x)$ is well defined on $\Omega_{\theta, \kappa}$ and it is analytic there.

REMARK 1.2. It is easy to check

$$
A \circ \chi=I_{E_{+}(\theta, \kappa)} \quad \text { and } \quad \chi \circ A=I_{\Omega_{\theta, \kappa}} .
$$

Under the above preparations we give the conditions for the other coefficients. A global analytic continuation property with respect to $x$-variable is required:

(A2) $\beta(x, \varepsilon), \gamma(x, \varepsilon)$ and $f(x, \varepsilon)$ can be continued analytically to $\Omega_{\theta, \kappa} \times\{\varepsilon \in C ;|\varepsilon| \leq$ $c$ \} for some $c>0$. Moreover, they satisfy the following estimates there:

$$
\begin{gathered}
\sup _{x \in \Omega_{\theta, \kappa},|\varepsilon| \leq c}\left|\frac{\beta(x, \varepsilon)}{\alpha(x)}\right|<\infty ; \\
\sup _{x \in \Omega_{\theta, \kappa},|\varepsilon| \leq c}|\gamma(x, \varepsilon)|<\infty ; \\
\max _{|\varepsilon| \leq c}|f(x, \varepsilon)| \leq C \exp [\delta|A(x)|], \quad x \in \Omega_{\theta, \kappa},
\end{gathered}
$$

where $C$ and $\delta$ are some positive constants independent of $x \in \Omega_{\theta, \kappa}$ and $\varepsilon$ with $|\varepsilon| \leq c$.

Let us state the main result in this paper.

THEOREM 1.5. Under assumptions (A1) and (A2) the divergent solution $u(x, \varepsilon)$ of (1.10) is Borel summable in the direction $\theta$. 
It should be remarked that the existence of the Borel sum, which is a local solution, is ensured by the global conditions such as (A1) and (A2).

To end this section, we give some simple examples of $\chi(\xi)$ and $A(x)$.

EXAMPLE 1.1. (1) When $\alpha(x) \equiv \mathrm{a}(\neq 0)$, we have $\chi(\xi)=-\mathrm{a} \xi$. Hence, (A1) is always satisfied for all $\theta$.

(2) When $\alpha(x)=\mathrm{a}+\mathrm{b} x(\mathrm{a}, \mathrm{b} \neq 0)$, we have $\chi(\xi)=(\mathrm{a} / \mathrm{b})\left(e^{-\mathrm{b} \xi}-1\right)$. Hence, (A1) is always satisfied for all $\theta$.

(3) When $\alpha(x)=1+x^{2}$, we have $\chi(\xi)=\tan (-\xi)$. Hence, if $\theta \neq 0$, $\pi$, then (A1) is satisfied.

(4) When $\alpha(x)=e^{x}$, we have $\chi(\xi)=-\log (\xi+1)$. Hence, (A1) is satisfied for all $\theta$ except $\theta=\pi$.

(5) When $\alpha(x)=1 /(1+x)$, we have $\chi(\xi)=-1+\sqrt{1-2 \xi}$, which implies (A1) for all $\theta$ except $\theta=0$.

(6) When $\alpha(x)=(x+1)(x-1) / 2$, we have $\chi(\xi)=\left(1-e^{-\xi}\right) /\left(1+e^{-\xi}\right)$. Hence, if $\theta \neq \pi / 2,3 \pi / 2$, (A1) is satisfied.

(7) When $\alpha(x)=(x-c)^{2}(c \neq 0)$, it holds that $\chi(\xi)=c^{2} \xi /(c \xi-1)$. Therefore, if $\theta \neq \arg (1 / \mathrm{c}),(\mathrm{A} 1)$ is satisfied.

EXAMPLE 1.2 (cf. Example 1.1). (1) When $\alpha(x) \equiv \mathrm{a}$, we have $A(x)=-x /$ a.

(2) When $\alpha(x)=\mathrm{a}+\mathrm{b} x$, we have $A(x)=-(1 / \mathrm{b}) \log (1+(\mathrm{b} / \mathrm{a}) x)$.

(3) When $\alpha(x)=1+x^{2}$, we have $A(x)=-\arctan x$.

(4) When $\alpha(x)=e^{x}$, we have $A(x)=e^{-x}-1$.

(5) When $\alpha(x)=1 /(1+x)$, we have $A(x)=-x-x^{2} / 2$.

(6) When $\alpha(x)=(x+1)(x-1) / 2$, we have $A(x)=\log (-(x+1) /(x-1))$.

(7) When $\alpha(x)=(x-\mathrm{c})^{2}$, we have $A(x)=1 /(x-\mathrm{c})+1 / \mathrm{c}$.

1.4. Some remarks on related topics. As was mentioned in the beginning of this paper, our equation has $x=0$ as a regular point. In the case where $x=0$ is a singular point, we can see some intriguing results in [3] (regular singular) and [4] (irregular singular). We can see quite different phenomena from ours there.

The present paper gives one of the generalizations of Hibino [6], where the case $\alpha(x)=$ $\mathrm{a}+\mathrm{b} x$ ( $\mathrm{a}, \mathrm{b}$ : constants; $\mathrm{a} \neq 0), \gamma(x, \varepsilon) \equiv 0$ and $\beta(x, \varepsilon)$ is a polynomial with respect to $\varepsilon$, is dealt with in detail. Moreover, in [6] we investigated partial differential equations such as

$$
\varepsilon \frac{\partial}{\partial x} u(x, \varepsilon)+\varepsilon^{2} \frac{\partial}{\partial \varepsilon} u(x, \varepsilon)+u(x, \varepsilon)=f(x, \varepsilon) .
$$

Equation (1.21) also has a unique power series solution $u(x, \varepsilon) \in \mathcal{O}[R][[\varepsilon]]_{2}$. However, the conditions under which this $u(x, \varepsilon)$ is Borel summable are different altogether from those stated in Theorem 1.5.

On the existence of the Gevrey asymptotic solutions in narrow regions such as stated in Theorem 1.3, we can find some interesting results in Canalis-Durand et al. [5] and Sibuya [14], where more general systems of singularly perturbed nonlinear ordinary differential equations 
are studied. Also, in the theory of partial differential equations we can find similar studies in some articles, for example Lutz et al. [11] and Ōuchi [13]. First-order partial differential equations of the nilpotent type including (1.21) were also dealt with in [8], where the same result as Theorem 1.3 was proved.

2. Formal Borel transform of equations. In this section, we reduce the proof of Theorem 1.5 to that of a global solvability of the initial value problem of some convolution equation. First we give some preliminaries.

DEFINITION 2.1. For $u(x, \varepsilon)=\sum_{n=0}^{\infty} u_{n}(x) \varepsilon^{n} \in \mathcal{O}[R][[\varepsilon]]_{2}$, we define the convergent power series $\mathcal{B}(u)(x, \eta)$ in a neighborhood of $(x, \eta)=(0,0)$ by

$$
\mathcal{B}(u)(x, \eta)=\sum_{n=0}^{\infty} u_{n}(x) \frac{\eta^{n}}{n !} .
$$

We call $\mathcal{B}(u)(x, \eta)$ the formal Borel transform of $u(x, \varepsilon)$.

When we would like to check the Borel summability of a given divergent power series $u(x, \varepsilon)=\sum_{n=0}^{\infty} u_{n}(x) \varepsilon^{n} \in \mathcal{O}[R][[\varepsilon]]_{2}$, the following theorem plays a fundamental role in general.

THEOREM 2.1 (Lutz et al. [11] and Malgrange [12]). For a power series $u(x, \varepsilon)=$ $\sum_{n=0}^{\infty} u_{n}(x) \varepsilon^{n} \in \mathcal{O}[R][[\varepsilon]]_{2}$, let us put $v(x, \eta)=\mathcal{B}(u)(x, \eta)$. Then the following conditions (i) and (ii) are equivalent.

(i) $u(x, \varepsilon)$ is Borel summable in a direction $\theta$.

(ii) $v(x, \eta)$ can be continued analytically to $B\left(r_{0}\right) \times E_{+}\left(\theta, \kappa_{0}\right)$ for some $r_{0}>0$ and $\kappa_{0}>0$, and has the following exponential growth estimate for some positive constants $C$ and $\delta$ :

$$
\max _{|x| \leq r_{0}}|v(x, \eta)| \leq C e^{\delta|\eta|}, \quad \eta \in E_{+}\left(\theta, \kappa_{0}\right) .
$$

When condition (i) or (ii) (therefore both) is satisfied, the Borel sum $U(x, \varepsilon)$ of $u(x, \varepsilon)$ in the direction $\theta$ is given by

$$
U(x, \varepsilon)=\frac{1}{\varepsilon} \int_{\boldsymbol{R}_{+} e^{i \theta}} e^{-\eta / \varepsilon} v(x, \eta) d \eta .
$$

Thus, in order to prove Theorem 1.5, it is sufficient to prove that the formal Borel transform $v(x, \eta)=\mathcal{B}(u)(x, \eta)$ of the divergent solution $u(x, \varepsilon)$ satisfies the above condition (ii) under assumptions (A1) and (A2). In order to do that, first let us write down the equation which $\mathcal{B}(u)(x, \eta)$ should satisfy. By operating the formal Borel transform to (1.10), we see that $\mathcal{B}(u)(x, \eta)$ is a solution of the following equation:

$$
\begin{aligned}
\alpha(x) \int_{0}^{\eta} D_{x} v(x, t) d t & +\int_{0}^{\eta} \mathcal{B}(\beta)(x, \eta-t) D_{x} v(x, t) d t \\
& +v(x, \eta)+\int_{0}^{\eta} \mathcal{B}(\gamma)(x, \eta-t) v(x, t) d t=\mathcal{B}(f)(x, \eta),
\end{aligned}
$$


where $\mathcal{B}(\beta)(x, \eta), \mathcal{B}(\gamma)(x, \eta)$ and $\mathcal{B}(f)(x, \eta)$ are the formal Borel transforms of $\beta(x, \varepsilon)=$ $\sum_{n=1}^{\infty} \beta_{n}(x) \varepsilon^{n}, \gamma(x, \varepsilon)=\sum_{n=0}^{\infty} \gamma_{n}(x) \varepsilon^{n}$ and $f(x, \varepsilon)=\sum_{n=0}^{\infty} f_{n}(x) \varepsilon^{n}$, respectively, that is,

$$
\mathcal{B}(\beta)(x, \eta)=\sum_{n=1}^{\infty} \beta_{n}(x) \frac{\eta^{n}}{n !}, \quad \mathcal{B}(\gamma)(x, \eta)=\sum_{n=0}^{\infty} \gamma_{n}(x) \frac{\eta^{n}}{n !}, \quad \mathcal{B}(f)(x, \eta)=\sum_{n=0}^{\infty} f_{n}(x) \frac{\eta^{n}}{n !} .
$$

Equation (2.4) is obtained by applying the following equality:

$$
\begin{aligned}
\mathcal{B}\left(y^{m+n+1}\right)(\eta) & =\frac{1}{(m+n+1) !} \eta^{m+n+1}=B(m+1, n+1) \frac{\eta^{m+n+1}}{m ! n !} \quad \text { (Beta integral) } \\
& =\int_{0}^{1}(1-s)^{m} s^{n} d s \cdot \frac{\eta^{m+n+1}}{m ! n !}=\int_{0}^{\eta}(\eta-t)^{m} t^{n} d t \cdot \frac{1}{m ! n !} \\
& =\int_{0}^{\eta} \mathcal{B}\left(y^{m}\right)(\eta-t) \mathcal{B}\left(y^{n}\right)(t) d t .
\end{aligned}
$$

Furthermore, by operating $D_{\eta}$ to (2.4) from the left, we see that $\mathcal{B}(u)(x, \eta)$ is a solution of the following initial value problem:

$$
\begin{aligned}
L v(x, \eta)= & -\int_{0}^{\eta} \mathcal{B}(\beta)_{\eta}(x, \eta-t) v_{x}(x, t) d t-\mathcal{B}(\gamma)(x, 0) v(x, \eta) \\
& -\int_{0}^{\eta} \mathcal{B}(\gamma)_{\eta}(x, \eta-t) v(x, t) d t+g(x, \eta), \\
v(x, 0)= & f(x, 0)
\end{aligned}
$$

where $L$ is the first-order linear partial differential operator defined by

$$
L=\alpha(x) D_{x}+D_{\eta},
$$

and $g(x, \eta)=\mathcal{B}(f)_{\eta}(x, \eta)$. It is easy to prove that $\mathcal{B}(u)(x, \eta)$ is the unique locally holomorphic solution of (2.5). Hence, Theorem 1.5 will be proved by showing that the solution $v(x, \eta)$ of (2.5) satisfies condition (ii) in Theorem 2.1.

3. Proof of Theorem 1.5. Let us start the proof of Theorem 1.5.

PROOF OF THEOREM 1.5. First of all, we transform the convolution equation (2.5) into the integral equation. We apply the following formula. The solution $V(x, \eta)$ of the initial value problem of the following first-order linear partial differential equation

$$
\begin{gathered}
\left\{\alpha(x) D_{x}+D_{\eta}\right\} V(x, \eta)=k(x, \eta), \\
V(x, 0)=l(x)
\end{gathered}
$$

is given by

$$
V(x, \eta)=l(\chi(A(x)+\eta))+\int_{0}^{\eta} k(\chi(A(x)+\eta-t), t) d t .
$$


By (3.2), we see that (2.5) is equivalent to the following equation:

$$
\begin{aligned}
v(x, \eta)= & f(\chi(A(x)+\eta), 0)+\int_{0}^{\eta} g(\chi(A(x)+\eta-t), t) d t \\
& -\int_{0}^{\eta} \int_{0}^{t} \mathcal{B}(\beta)_{\eta}(\chi(A(x)+\eta-t), t-s) v_{x}(\chi(A(x)+\eta-t), s) d s d t \\
& -\int_{0}^{\eta} \mathcal{B}(\gamma)(\chi(A(x)+\eta-t), 0) v(\chi(A(x)+\eta-t), t) d t \\
& -\int_{0}^{\eta} \int_{0}^{t} \mathcal{B}(\gamma)_{\eta}(\chi(A(x)+\eta-t), t-s) v(\chi(A(x)+\eta-t), s) d s d t .
\end{aligned}
$$

Furthermore, let us transform the third term of the right-hand side. By using Fubini's theorem, we write $\int_{0}^{\eta} \int_{0}^{t} \cdots d s d t=\int_{0}^{\eta} \int_{s}^{\eta} \cdots d t d s$. Here we remark that

$$
\begin{aligned}
& \int_{s}^{\eta} \mathcal{B}(\beta)_{\eta}(\chi(A(x)+\eta-t), t-s) v_{x}(\chi(A(x)+\eta-t), s) d t \\
& \quad=\int_{s}^{\eta} \frac{1}{\alpha(\chi(A(x)+\eta-t))} \mathcal{B}(\beta)_{\eta}(\chi(A(x)+\eta-t), t-s) \frac{\partial}{\partial t} v(\chi(A(x)+\eta-t), s) d t .
\end{aligned}
$$

Therefore by an integration by parts and Fubini's theorem again, we see that (2.5) is equivalent to the following integral equation:

$$
v(x, \eta)=f(\chi(A(x)+\eta), 0)+\int_{0}^{\eta} g(\chi(A(x)+\eta-t), t) d t+\sum_{i=1}^{7} I_{i} v(x, \eta),
$$

where each integral operator $I_{i}$ is given by

$$
\begin{aligned}
I_{1} v(x, \eta)= & -\frac{1}{\alpha(x)} \int_{0}^{\eta} \mathcal{B}(\beta)_{\eta}(x, \eta-t) v(x, t) d t \\
I_{2} v(x, \eta)= & \int_{0}^{\eta} \frac{1}{\alpha(\chi(A(x)+\eta-t))} \mathcal{B}(\beta)_{\eta}(\chi(A(x)+\eta-t), 0) v(\chi(A(x)+\eta-t), t) d t \\
I_{3} v(x, \eta)= & \int_{0}^{\eta} \int_{0}^{t} \frac{1}{\alpha(\chi(A(x)+\eta-t))} \\
& \times \mathcal{B}(\beta)_{\eta \eta}(\chi(A(x)+\eta-t), t-s) v(\chi(A(x)+\eta-t), s) d s d t \\
I_{4} v(x, \eta)= & -\int_{0}^{\eta} \int_{0}^{t} \frac{\alpha^{\prime}(\chi(A(x)+\eta-t))}{\alpha(\chi(A(x)+\eta-t))} \\
& \times \mathcal{B}(\beta)_{\eta}(\chi(A(x)+\eta-t), t-s) v(\chi(A(x)+\eta-t), s) d s d t \\
I_{5} v(x, \eta)= & \int_{0}^{\eta} \int_{0}^{t} \mathcal{B}(\beta)_{x \eta}(\chi(A(x)+\eta-t), t-s) v(\chi(A(x)+\eta-t), s) d s d t \\
I_{6} v(x, \eta)= & -\int_{0}^{\eta} \mathcal{B}(\gamma)(\chi(A(x)+\eta-t), 0) v(\chi(A(x)+\eta-t), t) d t \\
I_{7} v(x, \eta)= & -\int_{0}^{\eta} \int_{0}^{t} \mathcal{B}(\gamma)_{\eta}(\chi(A(x)+\eta-t), t-s) v(\chi(A(x)+\eta-t), s) d s d t .
\end{aligned}
$$


In order to prove that the solution $v(x, \eta)$ of (3.3) satisfies condition (ii) in Theorem 2.1, we employ the iteration method. Let us define $\left\{v_{n}(x, \eta)\right\}_{n=0}^{\infty}$ inductively as follows:

$$
\begin{gathered}
v_{0}(x, \eta)=f(\chi(A(x)+\eta), 0)+\int_{0}^{\eta} g(\chi(A(x)+\eta-t), t) d t \\
v_{n+1}(x, \eta)=v_{0}(x, \eta)+\sum_{i=1}^{7} I_{i} v_{n}(x, \eta) \quad(n \geq 0) .
\end{gathered}
$$

Next, we define $\left\{w_{n}(x, \eta)\right\}_{n=0}^{\infty}$ by $w_{0}(x, \eta)=v_{0}(x, \eta)$ and $w_{n}(x, \eta)=v_{n}(x, \eta)-v_{n-1}(x, \eta)$ $(n \geq 1)$, and define $\left\{W_{n}(x, \eta, t)\right\}_{n=0}^{\infty}$ by

$$
W_{n}(x, \eta, t)=w_{n}(\chi(A(x)+\eta-t), t) .
$$

Here we break the proof, and provide the notation needed in stating the key lemma later. Since $A(0)=0$, we can take $r_{0}>0$ and $\kappa_{0}>0$ such that

$$
\left\{A(x)+\zeta ;|x| \leq r_{0}, \zeta \in E_{+}\left(\theta, \kappa_{0}\right)\right\} \subset E_{+}(\theta, \kappa),
$$

where $\kappa>0$ is the constant given in assumption (A1). So let us define $\tilde{\beta}(x, \zeta, \varepsilon), \tilde{\gamma}(x, \zeta, \varepsilon)$ and $\mathcal{A}(x, \zeta)$ as follows:

$$
\begin{aligned}
\tilde{\beta}(x, \zeta, \varepsilon) & =\beta(\chi(A(x)+\zeta), \varepsilon), \\
\tilde{\gamma}(x, \zeta, \varepsilon) & =\gamma(\chi(A(x)+\zeta), \varepsilon), \\
\mathcal{A}(x, \zeta) & =\frac{1}{\alpha(\chi(A(x)+\zeta))} .
\end{aligned}
$$

Then it follows from the assumptions of Theorem 1.5 and (3.7) that $\tilde{\beta}(x, \zeta, \varepsilon)$ and $\tilde{\gamma}(x, \zeta, \varepsilon)$ are holomorphic on $\left\{x \in \boldsymbol{C} ;|x| \leq r_{0}\right\} \times E_{+}\left(\theta, \kappa_{0}\right) \times\{\varepsilon \in \boldsymbol{C} ;|\varepsilon| \leq c\}$, and that $\mathcal{A}(x, \zeta)$ is holomorphic on $\left\{x \in C ;|x| \leq r_{0}\right\} \times E_{+}\left(\theta, \kappa_{0}\right)$. Moreover, it holds that

$$
\sup _{|x| \leq r_{0}, \zeta \in E_{+}\left(\theta, \kappa_{0}\right),|\varepsilon| \leq c}|\mathcal{A}(x, \zeta) \tilde{\beta}(x, \zeta, \varepsilon)|<\infty
$$

and

$$
\sup _{|x| \leq r_{0}, \zeta \in E_{+}\left(\theta, \kappa_{0}\right),|\varepsilon| \leq c}|\tilde{\gamma}(x, \zeta, \varepsilon)|<\infty .
$$

Next we define $\mathcal{B}(\tilde{\beta})(x, \zeta, \eta)$ and $\mathcal{B}(\tilde{\gamma})(x, \zeta, \eta)$ by

$$
\mathcal{B}(\tilde{\beta})(x, \zeta, \eta)=\mathcal{B}(\beta)(\chi(A(x)+\zeta), \eta) \quad\left(=\sum_{n=1}^{\infty} \beta_{n}(\chi(A(x)+\zeta)) \frac{\eta^{n}}{n !}\right)
$$

and

$$
\mathcal{B}(\tilde{\gamma})(x, \zeta, \eta)=\mathcal{B}(\gamma)(\chi(A(x)+\zeta), \eta) \quad\left(=\sum_{n=0}^{\infty} \gamma_{n}(\chi(A(x)+\zeta)) \frac{\eta^{n}}{n !}\right)
$$


respectively. Then it follows from (3.11), (3.12) and Cauchy's integral formula that $\mathcal{B}(\tilde{\beta})(x, \zeta, \eta)$ and $\mathcal{B}(\tilde{\gamma})(x, \zeta, \eta)$ are holomorphic on $\left\{x \in \boldsymbol{C} ;|x| \leq r_{0}\right\} \times E_{+}\left(\theta, \kappa_{0}\right) \times \boldsymbol{C}$ and that there exist some positive constants $M$ and $\delta_{0}$ satisfying

$$
\begin{gathered}
\sup _{|x| \leq r_{0}, \zeta \in E_{+}\left(\theta, \kappa_{0}\right)}\left|\mathcal{A}(x, \zeta) \mathcal{B}(\tilde{\beta})_{\eta}(x, \zeta, \eta)\right| \leq M e^{\delta_{0}|\eta|}, \quad \eta \in \boldsymbol{C}, \\
\sup _{|x| \leq r_{0}, \zeta \in E_{+}\left(\theta, \kappa_{0}\right)}\left|\mathcal{A}(x, \zeta) \mathcal{B}(\tilde{\beta})_{\eta \eta}(x, \zeta, \eta)\right| \leq M e^{\delta_{0}|\eta|}, \quad \eta \in \boldsymbol{C}, \\
\sup _{|x| \leq r_{0}, \zeta \in E_{+}\left(\theta, \kappa_{0}^{\prime}\right)}\left|\frac{\partial}{\partial \zeta}\left\{\mathcal{A}(x, \zeta) \mathcal{B}(\tilde{\beta})_{\eta}(x, \zeta, \eta)\right\}\right| \leq M e^{\delta_{0}|\eta|}, \quad \eta \in \boldsymbol{C}, \\
\sup _{|x| \leq r_{0}, \zeta \in E_{+}\left(\theta, \kappa_{0}\right)}|\mathcal{B}(\tilde{\gamma})(x, \zeta, \eta)| \leq M e^{\delta_{0}|\eta|}, \quad \eta \in \boldsymbol{C}, \\
\sup _{|x| \leq r_{0}, \zeta \in E_{+}\left(\theta, \kappa_{0}\right)}\left|\mathcal{B}(\tilde{\gamma})_{\eta}(x, \zeta, \eta)\right| \leq M e^{\delta_{0}|\eta|}, \quad \eta \in \boldsymbol{C},
\end{gathered}
$$

where $\kappa_{0}^{\prime}=\kappa_{0} / 2$.

Finally, we give the following definition.

DEFINITION 3.1 .

(1) For $\lambda \geq 0$ and $\rho>0, U_{\rho}[0, \lambda]$ denotes the $\rho$-neighborhood of $[0, \lambda]$ in $\boldsymbol{C}$. Precisely,

$$
U_{\rho}[0, \lambda]=\{\tau \in \boldsymbol{C} ; \operatorname{dist}(\tau,[0, \lambda])<\rho\} .
$$

(2) For $\eta \in \boldsymbol{C}$, we define the function $G^{\eta}(\tau)$ by

$$
G^{\eta}(\tau)=\tau e^{i \arg (\eta)}, \quad \tau \in \boldsymbol{C},
$$

and define $G^{\eta}$ and $G_{\rho}^{\eta}$ as follows:

$$
\begin{aligned}
G^{\eta} & =\left\{G^{\eta}(R) \in C ; 0 \leq R \leq|\eta|\right\}, \\
G_{\rho}^{\eta} & =\left\{G^{\eta}(\tau) \in C ; \tau \in U_{\rho}[0,|\eta|]\right\} .
\end{aligned}
$$

We remark that $G^{\eta}$ is the segment from 0 to $\eta$ and that $G_{\rho}^{\eta}$ is the $\rho$-neighborhood of $G^{\eta}$.

Under these preparations let us take a monotonically decreasing positive sequence $\left\{\rho_{n}\right\}_{n=0}^{\infty}$ satisfying

$$
\tilde{\kappa}=\kappa_{0}^{\prime}-\sum_{n=0}^{\infty} \rho_{n}>0 .
$$

Then we obtain the following lemma.

LEMMA 3.1. $W_{n}(x, \eta, t)$ is continued analytically to $\left\{(x, \eta, t) ;|x| \leq r_{0}, \eta \in\right.$ $\left.E_{+}\left(\theta, \kappa_{0}^{\prime}-\sum_{j=0}^{n} \rho_{j}\right), t \in G_{\rho_{n}}^{\eta}\right\}$. Moreover, on $\left\{(x, \eta, t) ;|x| \leq r_{0}, \eta \in E_{+}\left(\theta, \kappa_{0}^{\prime}-\right.\right.$ $\left.\left.\sum_{j=0}^{n} \rho_{j}\right), t \in G^{\eta}\right\}$ we have the following estimate. For some positive constant $C_{1}$,

$$
\left|W_{n}\left(x, \eta, G^{\eta}(R)\right)\right| \leq C_{1} e^{\delta_{1}|\eta|}(3 M)^{n} \sum_{k=n}^{2 n}\left(\begin{array}{c}
n \\
k-n
\end{array}\right) \frac{R^{k}}{k !}, \quad 0 \leq R \leq|\eta|,
$$


where $\delta_{1}=\max \left\{\delta, \delta_{0}\right\}$ ( $\delta$ is the constant given in $(1.20)$ ).

We prove Lemma 3.1 in Section 4. For the present, we admit it and let us continue the proof of Theorem 1.5.

It follows from Lemma 3.1 that $w_{n}(x, \eta)\left(=W_{n}(x, \eta, \eta)\right)$ is continued analytically to $B\left(r_{0}\right) \times E_{+}\left(\theta, \kappa_{0}^{\prime}-\sum_{j=0}^{n} \rho_{j}\right)$ with the estimate

$$
\begin{aligned}
\left|w_{n}(x, \eta)\right| & =\left|W_{n}\left(x, \eta, G^{\eta}(|\eta|)\right)\right| \\
& \leq C_{1} e^{\delta_{1}|\eta|}(3 M)^{n} \sum_{k=n}^{2 n}\left(\begin{array}{c}
n \\
k-n
\end{array}\right) \frac{|\eta|^{k}}{k !} \\
& =C_{1} e^{\delta_{1}|\eta|}(3 M)^{n} \frac{1}{2 \pi i} \oint_{|z|=p}(1+z)^{n} e^{z|\eta|} \frac{1}{z^{2 n+1}} d z,
\end{aligned}
$$

where $p$ is an arbitrary positive number. Hence, by taking a suitably large $p$, we obtain on $B\left(r_{0}\right) \times E_{+}(\theta, \tilde{\kappa})$ that

$$
\begin{aligned}
\sum_{n=0}^{\infty}\left|w_{n}(x, \eta)\right| & \leq C_{1} e^{\delta_{1}|\eta|} \sum_{n=0}^{\infty}(3 M)^{n} \cdot \frac{1}{2 \pi i} \oint_{|z|=p}(1+z)^{n} e^{z|\eta|} \frac{1}{z^{2 n+1}} d z \\
& =C_{1} e^{\delta_{1}|\eta|} \frac{\mathcal{M}_{+} e^{\mathcal{M}_{+}|\eta|}-\mathcal{M}_{-} e^{\mathcal{M}_{-}|\eta|}}{\mathcal{M}_{+}-\mathcal{M}_{-}} \\
& \leq \frac{C_{1} \mathcal{M}_{+}}{\sqrt{9 M^{2}+12 M}} e^{\left(\delta_{1}+\mathcal{M}_{+}\right)|\eta|}
\end{aligned}
$$

where $\mathcal{M}_{ \pm}=(1 / 2)\left(3 M \pm \sqrt{9 M^{2}+12 M}\right)$. This shows that $v_{n}(x, \eta)\left(=\sum_{k=0}^{n} w_{k}(x, \eta)\right)$ converges to the solution $V(x, \eta)$ of (3.3) uniformly on $B\left(r_{0}\right) \times E_{+}(\theta, \tilde{\kappa})$. Consequently, $V(x, \eta)$ is an analytic continuation of $v(x, \eta)$, and it holds that

$$
\max _{|x| \leq r_{0}}|V(x, \eta)| \leq \frac{C_{1} \mathcal{M}_{+}}{\sqrt{9 M^{2}+12 M}} e^{\left(\delta_{1}+\mathcal{M}_{+}\right)|\eta|}, \quad \eta \in E_{+}(\theta, \tilde{\kappa}) .
$$

It follows from the above argument that $v(x, \eta)$ satisfies condition (ii) in Theorem 2.1. This completes the proof of Theorem 1.5.

4. Proof of Lemma 3.1. Let us prove Lemma 3.1. It is proved by the induction with respect to $n$.

Proof OF Lemma 3.1. First we consider the case $n=0$. By (3.4) and (3.6), we see that $W_{0}(x, \eta, t)$ has the following form:

$$
\begin{aligned}
W_{0}(x, \eta, t) & =f(\chi(A(x)+\eta), 0)+\int_{0}^{t} g(\chi(A(x)+\eta-s), s) d s \\
& \equiv J_{1}(x, \eta, t)+J_{2}(x, \eta, t) .
\end{aligned}
$$


Before proving the lemma for $W_{0}$, we remark the following. It follows from assumption (A2) and Cauchy's integral formula that $g(x, \eta)$ is analytic on $\Omega_{\theta, \kappa} \times \boldsymbol{C}$ with the estimate

$$
|g(x, \eta)| \leq C^{\prime} \exp [\delta|A(x)|] \cdot e^{\delta^{\prime}|\eta|}, \quad(x, \eta) \in \Omega_{\theta, \kappa} \times \boldsymbol{C},
$$

for some positive constants $C^{\prime}$ and $\delta^{\prime}$.

Now let us prove that $J_{1}(x, \eta, t)$ and $J_{2}(x, \eta, t)$ are well defined on $\{(x, \eta, t) ;|x| \leq$ $\left.r_{0}, \eta \in E_{+}\left(\theta, \kappa_{0}^{\prime}-\rho_{0}\right), t \in G_{\rho_{0}}^{\eta}\right\}$. Let $|x| \leq r_{0}, \eta \in E_{+}\left(\theta, \kappa_{0}^{\prime}-\rho_{0}\right), t \in G_{\rho_{0}}^{\eta}$, and let us write $t \in G_{\rho_{0}}^{\eta}$ as $t=G^{\eta}(\tau)\left(\tau \in U_{\rho_{0}}[0,|\eta|]\right)$.

On the well-definedness of $J_{1}\left(x, \eta, G^{\eta}(\tau)\right)$ : it is clear from the assumption (A2) and (3.7).

On the well-definedness of $J_{2}\left(x, \eta, G^{\eta}(\tau)\right)$ : in the integral expression of $J_{2}\left(x, \eta, G^{\eta}(\tau)\right)$, by taking a path of integration as

$$
s(\sigma)=\sigma e^{i \arg (\eta)} \quad(\sigma \in[0, \tau]),
$$

where $[0, \tau]$ is the segment from 0 to $\tau$, it holds that $\eta-s(\sigma) \in E_{+}\left(\theta, \kappa_{0}^{\prime}\right)\left(\subset E_{+}\left(\theta, \kappa_{0}\right)\right)$. Hence, it follows from (3.7) and the above remark that $J_{2}\left(x, \eta, G^{\eta}(\tau)\right)$ is well defined.

Therefore, $W_{0}(x, \eta, t)$ is well defined on $\left\{(x, \eta, t) ;|x| \leq r_{0}, t \in E_{+}\left(\theta, \kappa_{0}^{\prime}-\rho_{0}\right), t \in\right.$ $\left.G_{\rho_{0}}^{\eta}\right\}$. Moreover, on $\left\{(x, \eta, t) ;|x| \leq r_{0}, t \in E_{+}\left(\theta, \kappa_{0}^{\prime}-\rho_{0}\right), t \in G^{\eta}\right\}$ we have the following representation:

$$
\begin{aligned}
W_{0}\left(x, \eta, G^{\eta}(R)\right)= & f(\chi(A(x)+\eta), 0) \\
& +\int_{0}^{R} g\left(\chi\left(A(x)+\left(|\eta|-R_{1}\right) e^{i \arg (\eta)}\right), R_{1} e^{i \arg (\eta)}\right) e^{i \arg (\eta)} d R_{1} \\
\equiv & \mathcal{J}_{1}(x, \eta, R)+\mathcal{J}_{2}(x, \eta, R) .
\end{aligned}
$$

Let us estimate $\mathcal{J}_{1}(x, \eta, R)$ and $\mathcal{J}_{2}(x, \eta, R)$.

On $\mathcal{J}_{1}(x, \eta, R)$ : by $(1.20)$, we have

$$
\begin{aligned}
\left|\mathcal{J}_{1}(x, \eta, R)\right| & =|f(\chi(A(x)+\eta), 0)| \\
& \leq C \exp [\delta|A(\chi(A(x)+\eta))|]=C \exp [\delta|A(x)+\eta|] \\
& \leq C^{\prime \prime} e^{\delta|\eta|},
\end{aligned}
$$

where $C^{\prime \prime}=C \cdot \max _{|x| \leq r_{0}} \exp [\delta|A(x)|]$.

On $\mathcal{J}_{2}(x, \eta, R)$ : it follows from (4.1) that

$$
\left|g\left(\chi\left(A(x)+\left(|\eta|-R_{1}\right) e^{i \arg (\eta)}\right), R_{1} e^{i \arg (\eta)}\right)\right| \leq C^{\prime \prime \prime} e^{\delta|\eta|} e^{-\delta R_{1}} e^{\delta^{\prime} R_{1}}=C^{\prime \prime \prime} e^{\delta|\eta|} e^{-\left(\delta-\delta^{\prime}\right) R_{1}},
$$

where $C^{\prime \prime \prime}=C^{\prime} \cdot \max _{|x| \leq r_{0}} \exp [\delta|A(x)|]$. Here we may take $\delta>0$ so large that $\delta^{\prime \prime}=\delta-\delta^{\prime}>$ 0 . Hence, we obtain

$$
\left|\mathcal{J}_{2}(x, \eta, R)\right| \leq C^{\prime \prime \prime} e^{\delta|\eta|} \int_{0}^{R} e^{-\delta^{\prime \prime} R_{1}} d R_{1} \leq \frac{C^{\prime \prime \prime}}{\delta^{\prime \prime}} e^{\delta|\eta|} .
$$

By the above argument, it holds that

$$
\left|W_{0}\left(x, \eta, G^{\eta}(R)\right)\right| \leq C_{1} e^{\delta|\eta|} \leq C_{1} e^{\delta_{1}|\eta|},
$$

where $C_{1}=C^{\prime \prime}+C^{\prime \prime \prime} / \delta^{\prime \prime}$. Therefore, the lemma has been proved for $W_{0}$. 
Next, we assume that the claim of the lemma is proved up to $n$ and prove it for $n+1$. By (3.5) and (3.6) we have the following relation between $W_{n}$ and $W_{n+1}$ :

$$
W_{n+1}(x, \eta, t)=\sum_{i=1}^{6} \mathcal{I}_{i} W_{n}(x, \eta, t),
$$

where

$$
\begin{aligned}
\mathcal{I}_{1} W_{n}(x, \eta, t) & =I_{1} w_{n}(\chi(A(x)+\eta-t), t) \\
& =-\mathcal{A}(x, \eta-t) \int_{0}^{t} \mathcal{B}(\tilde{\beta})_{\eta}(x, \eta-t, t-s) W_{n}(x, \eta-t+s, s) d s, \\
\mathcal{I}_{2} W_{n}(x, \eta, t) & =I_{2} w_{n}(\chi(A(x)+\eta-t), t) \\
& =\int_{0}^{t} \mathcal{A}(x, \eta-s) \mathcal{B}(\tilde{\beta})_{\eta}(x, \eta-s, 0) W_{n}(x, \eta, s) d s, \\
\mathcal{I}_{3} W_{n}(x, \eta, t) & =I_{3} w_{n}(\chi(A(x)+\eta-t), t) \\
& =\int_{0}^{t} \int_{0}^{s} \mathcal{A}(x, \eta-s) \mathcal{B}(\tilde{\beta})_{\eta \eta}(x, \eta-s, s-y) W_{n}(x, \eta-s+y, y) d y d s, \\
\mathcal{I}_{4} W_{n}(x, \eta, t) & =I_{4} w_{n}(\chi(A(x)+\eta-t), t)+I_{5} w_{n}(\chi(A(x)+\eta-t), t) \\
& =-\left.\int_{0}^{t} \int_{0}^{s} \frac{\partial}{\partial \zeta}\left\{\mathcal{A}(x, \zeta) \mathcal{B}(\tilde{\beta})_{\eta}(x, \zeta, s-y)\right\}\right|_{\zeta=\eta-s} W_{n}(x, \eta-s+y, y) d y d s, \\
\mathcal{I}_{5} W_{n}(x, \eta, t) & =I_{6} w_{n}(\chi(A(x)+\eta-t), t) \\
& =-\int_{0}^{t} \mathcal{B}(\tilde{\gamma})(x, \eta-s, 0) W_{n}(x, \eta, s) d s, \\
\mathcal{I}_{6} W_{n}(x, \eta, t) & =I_{7} w_{n}(\chi(A(x)+\eta-t), t) \\
& =-\int_{0}^{t} \int_{0}^{s} \mathcal{B}(\tilde{\gamma})_{\eta}(x, \eta-s, s-y) W_{n}(x, \eta-s+y, y) d y d s .
\end{aligned}
$$

Let us prove that each $\mathcal{I}_{i} W_{n}(x, \eta, t)(i=1-6)$ is well defined on $\left\{(x, \eta, t) ;|x| \leq r_{0}, \eta \in\right.$ $\left.E_{+}\left(\theta, \kappa_{0}^{\prime}-\sum_{j=0}^{n+1} \rho_{j}\right), t \in G_{\rho_{n+1}}^{\eta}\right\}$ by taking suitable paths of integration. Let $|x| \leq r_{0}, \eta \in$ $E_{+}\left(\theta, \kappa_{0}^{\prime}-\sum_{j=0}^{n+1} \rho_{j}\right), t \in G_{\rho_{n+1}}^{\eta}$, and let us write $t \in G_{\rho_{n+1}}^{\eta}$ as $t=G^{\eta}(\tau)\left(\tau \in U_{\rho_{n+1}}[0,|\eta|]\right)$.

On $\mathcal{I}_{1} W_{n}\left(x, \eta, G^{\eta}(\tau)\right)$ : let us take a path of integration as (4.2). Then we have $\eta-$ $G^{\eta}(\tau)+s(\sigma) \in E_{+}\left(\theta, \kappa_{0}^{\prime}-\sum_{j=0}^{n} \rho_{j}\right)$ and $s(\sigma) \in G_{\rho_{n}}^{\eta-G^{\eta}(\tau)+s(\sigma)}$. Hence, $W_{n}(x, \eta-$ $\left.G^{\eta}(\tau)+s(\sigma), s(\sigma)\right)$ is well defined. It is obvious that $\mathcal{A}\left(x, \eta-G^{\eta}(\tau)\right)$ and $\mathcal{B}(\tilde{\beta})_{\eta}(x, \eta-$ $\left.G^{\eta}(\tau), G^{\eta}(\tau)-s(\sigma)\right)$ are well defined. Therefore, $\mathcal{I}_{1} W_{n}\left(x, \eta, G^{\eta}(\tau)\right)$ is well defined.

On $\mathcal{I}_{2} W_{n}\left(x, \eta, G^{\eta}(\tau)\right)$ and $\mathcal{I}_{5} W_{n}\left(x, \eta, G^{\eta}(\tau)\right)$ : let us take a path of integration as (4.2). Then we have $\eta \in E_{+}\left(\theta, \kappa_{0}^{\prime}-\sum_{j=0}^{n} \rho_{j}\right)$ and $s(\sigma) \in G_{\rho_{n}}^{\eta}$. Hence, $W_{n}(x, \eta, s(\sigma))$ is well defined. It is clear that $\mathcal{A}(x, \eta-s(\sigma)), \mathcal{B}(\tilde{\beta})_{\eta}(x, \eta-s(\sigma), 0)$ and $\mathcal{B}(\tilde{\gamma})(x, \eta-s(\sigma), 0)$ are well defined. Therefore, $\mathcal{I}_{2} W_{n}\left(x, \eta, G^{\eta}(\tau)\right)$ and $\mathcal{I}_{5} W_{n}\left(x, \eta, G^{\eta}(\tau)\right)$ are well defined. 
On $\mathcal{I}_{i} W_{n}\left(x, \eta, G^{\eta}(\tau)\right)(i=3,4,6)$ : we only state paths of integration. By taking paths of integration as

$$
\begin{array}{cc}
s(\sigma)=\sigma e^{i \arg (\eta)} & (\sigma \in[0, \tau]), \\
y(\lambda)=\lambda e^{i \arg (\eta)} \quad(\lambda \in[0, \sigma]),
\end{array}
$$

we see that all $\mathcal{I}_{i} W_{n}\left(x, \eta, G^{\eta}(\tau)\right)(i=3,4,6)$ are well defined.

Therefore, $W_{n+1}(x, \eta, t)$ is well defined on $\left\{(x, \eta, t) ;|x| \leq r_{0}, \eta \in E_{+}\left(\theta, \kappa_{0}^{\prime}-\right.\right.$ $\left.\left.\sum_{j=0}^{n+1} \rho_{j}\right), t \in G_{\rho_{n+1}}^{\eta}\right\}$. Moreover, on $\left\{(x, \eta, t) ;|x| \leq r_{0}, \eta \in E_{+}\left(\theta, \kappa_{0}^{\prime}-\sum_{j=0}^{n+1} \rho_{j}\right), t \in\right.$ $\left.G^{\eta}\right\}$ we have the following representations:

$$
\begin{aligned}
& \mathcal{I}_{1} W_{n}\left(x, \eta, G^{\eta}(R)\right)=-\mathcal{A}\left(x,(|\eta|-R) e^{i \arg (\eta)}\right) \\
& \times \int_{0}^{R} \mathcal{B}(\tilde{\beta})_{\eta}\left(x,(|\eta|-R) e^{i \arg (\eta)},\left(R-R_{1}\right) e^{i \arg (\eta)}\right) \\
& \times \mathcal{W}_{n}\left(x, \eta, R, R_{1}\right) e^{i \arg (\eta)} d R_{1}, \\
& \mathcal{I}_{2} W_{n}\left(x, \eta, G^{\eta}(R)\right)=\int_{0}^{R} \mathcal{A}\left(x,\left(|\eta|-R_{1}\right) e^{i \arg (\eta)}\right) \mathcal{B}(\tilde{\beta})_{\eta}\left(x,\left(|\eta|-R_{1}\right) e^{i \arg (\eta)}, 0\right) \\
& \times \mathcal{W}_{n}\left(x, \eta, R_{1}, R_{1}\right) e^{i \arg (\eta)} d R_{1}, \\
& \mathcal{I}_{3} W_{n}\left(x, \eta, G^{\eta}(R)\right)=\int_{0}^{R} \int_{0}^{R_{1}} \mathcal{A}\left(x,\left(|\eta|-R_{1}\right) e^{i \arg (\eta)}\right) \\
& \times \mathcal{B}(\tilde{\beta})_{\eta \eta}\left(x,\left(|\eta|-R_{1}\right) e^{i \arg (\eta)},\left(R_{1}-R_{2}\right) e^{i \arg (\eta)}\right) \\
& \times \mathcal{W}_{n}\left(x, \eta, R_{1}, R_{2}\right)\left\{e^{i \arg (\eta)}\right\}^{2} d R_{2} d R_{1}, \\
& \mathcal{I}_{4} W_{n}\left(x, \eta, G^{\eta}(R)\right)=-\int_{0}^{R} \int_{0}^{R_{1}} \frac{\partial}{\partial \zeta}\{\mathcal{A}(x, \zeta) \\
& \left.\times \mathcal{B}(\tilde{\beta})_{\eta}\left(x, \zeta,\left(R_{1}-R_{2}\right) e^{i \arg (\eta)}\right)\right\}\left.\right|_{\zeta=\left(|\eta|-R_{1}\right) e^{i \arg (\eta)}} \\
& \times \mathcal{W}_{n}\left(x, \eta, R_{1}, R_{2}\right)\left\{e^{i \arg (\eta)}\right\}^{2} d R_{2} d R_{1}, \\
& \mathcal{I}_{5} W_{n}\left(x, \eta, G^{\eta}(R)\right)=-\int_{0}^{R} \mathcal{B}(\tilde{\gamma})\left(x,\left(|\eta|-R_{1}\right) e^{i \arg (\eta)}, 0\right) \mathcal{W}_{n}\left(x, \eta, R_{1}, R_{1}\right) e^{i \arg (\eta)} d R_{1}, \\
& \mathcal{I}_{6} W_{n}\left(x, \eta, G^{\eta}(R)\right)=-\int_{0}^{R} \int_{0}^{R_{1}} \mathcal{B}(\tilde{\gamma})_{\eta}\left(x,\left(|\eta|-R_{1}\right) e^{i \arg (\eta)},\left(R_{1}-R_{2}\right) e^{i \arg (\eta)}\right) \\
& \times \mathcal{W}_{n}\left(x, \eta, R_{1}, R_{2}\right)\left\{e^{i \arg (\eta)}\right\}^{2} d R_{2} d R_{1},
\end{aligned}
$$

where

$$
\mathcal{W}_{n}(x, \eta, \mu, v)=W_{n}\left(x,(|\eta|-\mu+v) e^{i \arg (\eta)}, G^{\left.(|\eta|-\mu+v) e^{i \arg (\eta)}(v)\right) .}\right.
$$

Let us estimate each $\mathcal{I}_{i} W_{n}\left(x, \eta, G^{\eta}(R)\right)$.

On $\mathcal{I}_{1} W_{n}\left(x, \eta, G^{\eta}(R)\right)$ : it follows from the assumption of the induction that

$$
\left|\mathcal{W}_{n}\left(x, \eta, R, R_{1}\right)\right| \leq C_{1} e^{\delta_{1}|\eta|} e^{-\delta_{1} R} e^{\delta_{1} R_{1}}(3 M)^{n} \sum_{k=n}^{2 n}\left(\begin{array}{c}
n \\
k-n
\end{array}\right) \frac{R_{1}{ }^{k}}{k !} .
$$


Hence (3.15) and $\delta_{0} \leq \delta_{1}$ imply that

$$
\begin{aligned}
\left|\mathcal{I}_{1} W_{n}\left(x, \eta, G^{\eta}(R)\right)\right| & \leq C_{1} e^{\delta_{1}|\eta|}(3 M)^{n} M \sum_{k=n}^{2 n}\left(\begin{array}{c}
n \\
k-n
\end{array}\right) \int_{0}^{R} \frac{R_{1}{ }^{k}}{k !} d R_{1} \\
& =C_{1} e^{\delta_{1}|\eta|}(3 M)^{n} M \sum_{k=n+1}^{2 n+1}\left(\begin{array}{c}
n \\
k-1-n
\end{array}\right) \frac{R^{k}}{k !} .
\end{aligned}
$$

On $\mathcal{I}_{2} W_{n}\left(x, \eta, G^{\eta}(R)\right)$ : let us consider $R_{1}$ instead of $R$ in (4.6). Then we have

$$
\left|\mathcal{W}_{n}\left(x, \eta, R_{1}, R_{1}\right)\right| \leq C_{1} e^{\delta_{1}|\eta|}(3 M)^{n} \sum_{k=n}^{2 n}\left(\begin{array}{c}
n \\
k-n
\end{array}\right) \frac{R_{1}{ }^{k}}{k !} .
$$

Hence, we see by (3.15) and $\delta_{0} \leq \delta_{1}$ that $\mathcal{I}_{2} W_{n}\left(x, \eta, G^{\eta}(R)\right)$ has the same estimate as that of $\mathcal{I}_{1} W_{n}\left(x, \eta, G^{\eta}(R)\right)$. Similarly, we can obtain the same estimate for $\mathcal{I}_{5} W_{n}\left(x, \eta, G^{\eta}(R)\right)$. Therefore, it holds that

$$
\begin{aligned}
& \left|\mathcal{I}_{1} W_{n}\left(x, \eta, G^{\eta}(R)\right)\right|+\left|\mathcal{I}_{2} W_{n}\left(x, \eta, G^{\eta}(R)\right)\right|+\left|\mathcal{I}_{5} W_{n}\left(x, \eta, G^{\eta}(R)\right)\right| \\
& \quad \leq C_{1} e^{\delta_{1}|\eta|}(3 M)^{n+1} \sum_{k=n+1}^{2 n+1}\left(\begin{array}{c}
n \\
k-1-n
\end{array}\right) \frac{R^{k}}{k !} .
\end{aligned}
$$

On $\mathcal{I}_{3} W_{n}\left(x, \eta, G^{\eta}(R)\right)$ : it follows from the assumption of the induction that

$$
\left|\mathcal{W}_{n}\left(x, \eta, R_{1}, R_{2}\right)\right| \leq C_{1} e^{\delta_{1}|\eta|} e^{-\delta_{1} R_{1}} e^{\delta_{1} R_{2}}(3 M)^{n} \sum_{k=n}^{2 n}\left(\begin{array}{c}
n \\
k-n
\end{array}\right) \frac{R_{2}{ }^{k}}{k !} .
$$

Hence, (3.15) and $\delta_{0} \leq \delta_{1}$ imply that

$$
\begin{aligned}
\left|\mathcal{I}_{3} W_{n}\left(x, \eta, G^{\eta}(R)\right)\right| & \leq C_{1} e^{\delta_{1}|\eta|}(3 M)^{n} M \sum_{k=n}^{2 n}\left(\begin{array}{c}
n \\
k-n
\end{array}\right) \int_{0}^{R} \int_{0}^{R_{1}} \frac{R_{2}{ }^{k}}{k !} d R_{2} d R_{1} \\
& =C_{1} e^{\delta_{1}|\eta|}(3 M)^{n} M \sum_{k=n+2}^{2(n+1)}\left(\begin{array}{c}
n \\
k-2-n
\end{array}\right) \frac{R^{k}}{k !} .
\end{aligned}
$$

Similarly, we can prove that $\mathcal{I}_{4} W_{n}\left(x, \eta, G^{\eta}(R)\right)$ and $\mathcal{I}_{6} W_{n}\left(x, \eta, G^{\eta}(R)\right)$ have the same estimate as that of $\mathcal{I}_{3} W_{n}\left(x, \eta, G^{\eta}(R)\right)$. Therefore, it holds that

$$
\begin{gathered}
\left|\mathcal{I}_{3} W_{n}\left(x, \eta, G^{\eta}(R)\right)\right|+\left|\mathcal{I}_{4} W_{n}\left(x, \eta, G^{\eta}(R)\right)\right|+\left|\mathcal{I}_{6} W_{n}\left(x, \eta, G^{\eta}(R)\right)\right| \\
\leq C_{1} e^{\delta_{1}|\eta|}(3 M)^{n+1} \sum_{k=n+2}^{2(n+1)}\left(\begin{array}{c}
n \\
k-2-n
\end{array}\right) \frac{R^{k}}{k !} .
\end{gathered}
$$

Finally let us combine (4.7) and (4.8). Then we obtain

$$
\begin{aligned}
& \left|W_{n+1}\left(x, \eta, G^{\eta}(R)\right)\right| \\
& \quad \leq \sum_{i=1}^{6}\left|\mathcal{I}_{i} W_{n}\left(x, \eta, G^{\eta}(R)\right)\right|
\end{aligned}
$$




$$
\begin{aligned}
\leq & C_{1} e^{\delta_{1}|\eta|}(3 M)^{n+1} \\
& \times\left\{\frac{R^{n+1}}{(n+1) !}+\sum_{k=n+2}^{2 n+1}\left\{\left(\begin{array}{c}
n \\
k-1-n
\end{array}\right)+\left(\begin{array}{c}
n \\
k-2-n
\end{array}\right)\right\} \frac{R^{k}}{k !}+\frac{R^{2(n+1)}}{\{2(n+1)\} !}\right\} \\
= & C_{1} e^{\delta_{1}|\eta|}(3 M)^{n+1} \sum_{k=n+1}^{2(n+1)}\left(\begin{array}{c}
n+1 \\
k-(n+1)
\end{array}\right) \frac{R^{k}}{k !},
\end{aligned}
$$

which implies the lemma for $n+1$. The proof has been completed.

\section{Special cases.}

5.1. Necessary and sufficient condition. Theorem 1.5 gives only the sufficient condition on the Borel summability. When an equation has a restricted form, we can obtain the necessary and sufficient condition under which the divergent solution is Borel summable. Here we consider the case $\beta(x, \varepsilon) \equiv \gamma(x, \varepsilon) \equiv 0$. Moreover, we assume that $f(x, \varepsilon)$ is a polynomial with respect to $\varepsilon$-variable. Precisely, we consider the following equation:

$$
\alpha(x) \varepsilon D_{x} u(x, \varepsilon)+u(x, \varepsilon)=\sum_{l=0}^{k} f_{l}(x) \varepsilon^{l},
$$

where each $f_{l}(x)(l=0,1,2, \ldots, k)$ is holomorphic at $x=0$.

In order to state the theorem, we introduce the notation. We define the first-order differential operator $\Theta$ by

$$
\Theta=-\alpha(x) \frac{d}{d x},
$$

and define the function $\tilde{f}(x)$ by

$$
\tilde{f}(x)=\sum_{l=0}^{k} \Theta^{k-l} f_{l}(x) .
$$

Then we have the following theorem.

THEOREM 5.1. Let us assume (A1). Then the following two statements (1) and (2) are equivalent.

(1) The divergent solution $u(x, \varepsilon)$ of (5.1) is Borel summable in the direction $\theta$.

(2) $\tilde{f}(x)$ can be continued analytically to $\Omega_{\theta, \kappa}$, and it satisfies the following estimate for some positive constants $C$ and $\delta$ :

$$
|\tilde{f}(x)| \leq C \exp [\delta|A(x)|], \quad x \in \Omega_{\theta, \kappa} .
$$

PROOF. For $u(x, \varepsilon)=\sum_{n=0}^{\infty} u_{n}(x) \varepsilon^{n}$, let us define $\tilde{u}(x, \varepsilon)$ by

$$
u(x, \varepsilon)=\sum_{n=0}^{k-1} u_{n}(x) \varepsilon^{n}+\varepsilon^{k} \tilde{u}(x, \varepsilon) .
$$


We remark that $u(x, \varepsilon)$ is Borel summable if and only if $\tilde{u}(x, \varepsilon)$ is Borel summable. Moreover, we have the following relation between the Borel sum $U(x, \varepsilon)$ of $u(x, \varepsilon)$ and the Borel sum $\tilde{U}(x, \varepsilon)$ of $\tilde{u}(x, \varepsilon): U(x, \varepsilon)=\varepsilon^{k} \tilde{U}(x, \varepsilon)+\sum_{n=0}^{k-1} u_{n}(x) \varepsilon^{n}$. So let us write down the equation which $\tilde{u}(x, \varepsilon)$ should satisfy. By an easy calculation, it follows that $\tilde{u}(x, \varepsilon)$ satisfies the following equation:

$$
\alpha(x) \varepsilon D_{x} \tilde{u}(x, \varepsilon)+\tilde{u}(x, \varepsilon)=\tilde{f}(x) .
$$

Hence, by Theorem 1.5, if we assume the condition (2), we can obtain the Borel summability of $\tilde{u}(x, \varepsilon)$ in the direction $\theta$. This shows (2) $\Rightarrow(1)$.

In order to prove (1) $\Rightarrow(2)$, let us adopt Theorem 2.1. First we remark that the formal Borel transform $\tilde{v}(x, \varepsilon)=\mathcal{B}(\tilde{u})(x, \varepsilon)$ of $\tilde{u}(x, \varepsilon)$ can be written explicitly as follows:

$$
\tilde{v}(x, \eta)=\tilde{f}(\chi(A(x)+\eta)) .
$$

Therefore, it follows from (1) and Theorem 2.1 that $\tilde{V}(\eta)=\tilde{v}(0, \eta)=\tilde{f}(\chi(\eta))$ can be continued analytically to $E_{+}\left(\theta, \kappa_{0}\right)$ for some $\kappa_{0}>0$, and that there exist some positive constants $C$ and $\delta$ satisfying

$$
|\tilde{V}(\eta)| \leq C e^{\delta|\eta|}, \quad \eta \in E_{+}\left(\theta, \kappa_{0}\right) .
$$

Here we may take $\kappa>0$ so small that $\kappa \leq \kappa_{0}$. Then $\tilde{f}(x)=\tilde{V}(A(x))$ can be continued analytically to $\Omega_{\theta, \kappa}$, and it holds that $|\tilde{f}(x)|=|\tilde{V}(A(x))| \leq C \exp [\delta|A(x)|]$ for $x \in \Omega_{\theta, \kappa}$. Consequently, (1) $\Rightarrow(2)$ has been proved.

REMARK 5.1. In (5.1), each $u_{n}(x)(n=0,1,2, \ldots)$ can be written explicitly as

$$
u_{n}(x)= \begin{cases}\sum_{l=0}^{n} \Theta^{n-l} f_{l}(x) & (n=0,1,2, \ldots, k-1), \\ \sum_{l=0}^{k} \Theta^{n-l} f_{l}(x) & (n=k, k+1, \ldots) .\end{cases}
$$

Therefore, it follows from the above proof that the Borel sum $U(x, \varepsilon)$ (in the direction $\theta$ ) of $u(x, \varepsilon)$ has the following form:

$$
U(x, \varepsilon)=\varepsilon^{k-1} \int_{\boldsymbol{R}_{+} e^{i \theta}} e^{-\eta / \varepsilon} \tilde{f}(\chi(A(x)+\eta)) d \eta+\sum_{n=0}^{k-1}\left\{\sum_{l=0}^{n} \Theta^{n-l} f_{l}(x)\right\} \varepsilon^{n} .
$$

Theorem 5.1 does not require the analytic continuation property for $f(x, \varepsilon)$ itself, as will be shown in the following example.

EXAMPLE 5.1. Let $\theta=0$.

(1) We consider the following equation:

$$
\begin{aligned}
-(1+x) \varepsilon D_{x} u(x, \varepsilon)+u(x, \varepsilon) & =f(x, \varepsilon)=f_{0}(x)+f_{1}(x) \varepsilon \\
& =\frac{1}{1-x}+\left\{\sqrt{1+\log (1+x)}-\frac{1+x}{(1-x)^{2}}\right\} \varepsilon .
\end{aligned}
$$


Equation (5.9) has the divergent solution

$$
\begin{aligned}
u(x, \varepsilon)= & \frac{1}{1-x}+\sqrt{1+\log (1+x)} \varepsilon+\frac{1}{2} \frac{1}{\sqrt{1+\log (1+x)}} \varepsilon^{2} \\
& +\sum_{n=3}^{\infty} \frac{(-1)^{n}}{2^{n-1}}(2 n-5) ! !\{1+\log (1+x)\}^{-(n-3 / 2)} \varepsilon^{n} .
\end{aligned}
$$

$\alpha(x)=-(1+x)$ implies $\chi(\xi)=e^{\xi}-1$ and $A(x)=\log (1+x)$. Hence, condition (A1) is satisfied. Since $f(x, \varepsilon)$ cannot be continued analytically along the positive real axis with respect to $x$, it does not satisfy condition (A2), but $\tilde{f}(x)=(1+x) f_{0}^{\prime}(x)+f_{1}(x)=\sqrt{1+\log (1+x)}$ clearly satisfies condition (2) in Theorem 5.1. Therefore, the above $u(x, \varepsilon)$ is Borel summable in the direction 0 and its Borel sum $U(x, \varepsilon)$ in the direction 0 is given by

$$
U(x, \varepsilon)=\int_{0}^{\infty} e^{-\eta / \varepsilon} \sqrt{1+\log (1+x)+\eta} d \eta+\frac{1}{1-x} .
$$

(2) Let us consider the following equation:

$$
-e^{-x} \varepsilon D_{x} u(x, \varepsilon)+u(x, \varepsilon)=f(x, \varepsilon)=f_{0}(x)+f_{1}(x) \varepsilon+f_{2}(x) \varepsilon^{2}
$$

$$
=\frac{1}{1-x}+\frac{e^{-x}}{(1-x)^{2}} \varepsilon+\left\{\frac{1}{e^{x}+1}-\frac{2 e^{-2 x}(1+x)}{(1-x)^{3}}\right\} \varepsilon^{2} .
$$

Equation (5.10) has the divergent solution

$$
u(x, \varepsilon)=\frac{1}{1-x}+\frac{2 e^{-x}}{(1-x)^{2}} \varepsilon+\sum_{n=2}^{\infty} \frac{(-1)^{n}(n-2) !}{\left(e^{x}+1\right)^{n-1}} \varepsilon^{n} .
$$

Since $\alpha(x)=-e^{-x}$, we have $\chi(\xi)=\log (1+\xi)$ and $A(x)=e^{x}-1$. Hence, condition (A1) is satisfied. In this case also, $f(x, \varepsilon)$ itself does not satisfy condition (A2), but $\tilde{f}(x)=$ $e^{-x}(d / d x)\left\{e^{-x} f_{0}^{\prime}(x)\right\}+e^{-x} f_{1}^{\prime}(x)+f_{2}(x)=1 /\left(e^{x}+1\right)$ satisfies condition (2) in Theorem 5.1. Therefore, $u(x, \varepsilon)$ is Borel summable in the direction 0 and its Borel sum $U(x, \varepsilon)$ in the direction 0 is given by

$$
U(x, \varepsilon)=\varepsilon \int_{0}^{\infty} e^{-\eta / \varepsilon} \frac{1}{1+e^{x}+\eta} d \eta+\frac{1}{1-x}+\frac{2 e^{-x}}{(1-x)^{2}} \varepsilon .
$$

Here we deal with the case $k=0$; that is, we consider the following equation:

$$
\alpha(x) \varepsilon D_{x} u(x, \varepsilon)+u(x, \varepsilon)=f(x) .
$$

Furthermore we assume that the existence domain of $f(x)$ is bounded. By using Theorem 5.1, let us try investigating the Borel summability of $u(x, \varepsilon)$ concretely.

EXAMPLE 5.2. (1) $\alpha(x)=-(1+x)$ : in this case we have $\chi(\xi)=e^{\xi}-1$, and assumption (A1) is satisfied for all $\theta$. If $0 \leq \theta<\pi / 2$ or $3 \pi / 2<\theta<2 \pi$, then the region $\Omega_{\theta, \kappa}$ is unbounded for all $\kappa>0$. Hence, $u(x, \varepsilon)$ is never Borel summable in these directions $\theta$. If $\pi / 2 \leq \theta \leq 3 \pi / 2$, we see that for any $\rho>0$ there exists some $\kappa>0$ such that $\Omega_{\theta, \kappa} \subset$ 
$\{x \in \boldsymbol{C} ;|x| \leq 2+\rho\}$. Therefore, if $f(x)$ is holomorphic on $\{x \in \boldsymbol{C} ;|x| \leq 2+\rho\}$ for some $\rho>0$, then $u(x, \varepsilon)$ is always Borel summable in the direction $\theta$ satisfying $\pi / 2 \leq \theta \leq 3 \pi / 2$.

(2) $\alpha(x)=-e^{-x}$ : in this case we have $\chi(\xi)=\log (1+\xi)$. Hence, assumption (A1) is satisfied for all $\theta$ except $\theta=\pi$, and the region $\Omega_{\theta, \kappa}$ is always unbounded for all $\kappa>0$ and $\theta \neq \pi$. Therefore, $u(x, \varepsilon)$ is by no means Borel summable in the direction $\theta$ satisfying $\theta \neq \pi$.

(3) $\alpha(x)=(x-1)^{2}$ : in this case it holds that $\chi(\xi)=\xi /(\xi-1)$. Hence, if $\theta \neq 0$, then condition (A1) is satisfied. Moreover, if $\theta \neq 0$, we see that for any $\rho>0$ there exists some $\kappa>0$ such that $\Omega_{\theta, \kappa} \subset\left\{x \in \boldsymbol{C} ;|x| \leq 1+\rho+1 / \min _{\xi \in \boldsymbol{R}_{+} e^{i \theta}}|\xi-1|\right\}$. Therefore if $f(x)$ is holomorphic on $\left\{x \in \boldsymbol{C} ;|x| \leq 1+\rho+1 / \min _{\xi \in \boldsymbol{R}_{+} e^{i \theta}}|\xi-1|\right\}$ for some $\rho>0$, then $u(x, \varepsilon)$ is always Borel summable in all directions $\theta \neq 0$.

5.2. Another example. Both in Theorem 1.5 and in Theorem 5.1 we imposed assumption (A1) for the coefficient $\alpha(x)$. In the case where (A1) is not satisfied, it is in general difficult to give conditions for the other coefficients because we cannot define the region $\Omega_{\theta, \kappa}$. However, it is within possibility that the divergent solution is Borel summable even if (A1) is not fulfilled. Last of all, we give such an example.

Let us consider the following equation:

$$
-\left(1+x^{2}\right) \varepsilon D_{x} u(x, \varepsilon)+u(x, \varepsilon)=\frac{1}{1-\arctan x} .
$$

Equation (5.12) has the divergent solution

$$
u(x, \varepsilon)=\sum_{n=0}^{\infty} \frac{n !}{(1-\arctan x)^{n+1}} \varepsilon^{n} .
$$

Since $\alpha(x)=-\left(1+x^{2}\right)$, we have $\chi(\xi)=\tan \xi$. Hence, condition (A1) is not satisfied for $\theta=0, \pi$. However, we can prove that the above $u(x, \varepsilon)$ is Borel summable in the direction $\pi$ by means of the formula $\mathcal{B}(u)(x, \eta)=1 /(1-\arctan x-\eta)$. Moreover, we see that $u(x, \varepsilon)$ is Borel summable in all directions $\theta$ except $\theta=0$, and that it is never Borel summable in the direction 0 .

\section{REFERENCES}

[ 1 ] W. BALSER, Formal power series and linear systems of meromorphic ordinary differential equations, Universitext, Springer, New York, 2000.

[2] W. BALSER, From divergent power series to analytic functions, Theory and application of multisummable power series, Lecture Notes in Math. 1582, Springer, Berlin, 1994.

[ 3 ] W. BAlser And V. Kostov, Singular perturbation of linear systems with a regular singularity, J. Dynam. Control Systems 8 (2002), 313-322.

[ 4 ] W. BALSER AND J. MOZO-FERnÁNDEZ, Multisummability of formal solutions of singular perturbation problems, J. Differential Equations 183 (2002), 526-545.

[ 5 ] M. Canalis-Durand, J. P. Ramis, R. SchäFKe And Y. SibuYA, Gevrey solutions of singularly perturbed differential equations, J. Reine Angew. Math. 518 (2000), 95-129.

[6] M. Hibino, Borel summability of divergent solutions for singular first order linear partial differential equations with polynomial coefficients, J. Math. Sci. Univ. Tokyo 10 (2003), 279-309. 
[ 7 ] M. Hibino, Divergence property of formal solutions for singular first order linear partial differential equations, Publ. Res. Inst. Math. Sci. 35 (1999), 893-919.

[ 8 ] M. Hibino, Gevrey asymptotic theory for singular first order linear partial differential equations of nilpotent type I, Commun. Pure Appl. Anal. 2 (2003), 211-231.

[9] M. Hibino, Gevrey asymptotic theory for singular first order linear partial differential equations of nilpotent type II, Publ. Res. Inst. Math. Sci. 37 (2001), 579-614.

[10] M. Hibino, Gevrey theory for singular first order partial differential equations in complex domain, Doctoral thesis, Graduate School of Mathematics, Nagoya University, 2002.

[11] D. A. Lutz, M. Miyake And R. SchäfKe, On the Borel summability of divergent solutions of the heat equation, Nagoya Math. J. 154 (1999), 1-29.

[12] B. Malgrange, Sommation des séries divergentes, Exposition. Math. 13 (1995), 163-222.

[13] S. ÖUCHI, Genuine solutions and formal solutions with Gevrey type estimates of nonlinear partial differential equations, J. Math. Sci. Univ. Tokyo 2 (1995), 375-417.

[14] Y. SiBUYA, The Gevrey asymptotics in the case of singular perturbations, J. Differential Equations 165 (2000), 255-314.

Research Fellow of the Japan Society for the Promotion of Science DEPARTMENT OF MATHEMATICS

Meijo University

SHIOGAMAGUCHI, TEMPAKU, NAGOYA

AICHI 468-8502

JAPAN

E-mail address: hibinom@ccmfs.meijo-u.ac.jp 